introduction of IC, though at a slower rate than if IC had not existed.

Conclusion The introduction of a national target resulted in a reduction of delayed days. After factoring out this reduction, IC was responsible for a further reduction in delayed days of $18 \%$. The rate of days delayed continued to increase over time after the introduction of IC, though at a slower rate.

\section{P53 THE ASSOCIATIONS BETWEEN COMMON MENTAL DISORDERS (ANXIETY AND DEPRESSION) AND SOCIO- ECONOMIC AND DEMOGRAPHIC FACTORS IN TWO RUSSIAN CITIES}

${ }^{1} \mathrm{~S}$ Cook*, ${ }^{2} \mathrm{AV}$ Kudryavtsev, ${ }^{1} \mathrm{~N}$ Bobrova, ${ }^{3,4} \mathrm{~S}$ Malyutina, ${ }^{1,5} \mathrm{DA}$ Leon. ${ }^{1}$ Department of NonCommunicable Disease Epidemiology, London School of Hygiene and Tropical Medicine, London, UK; ${ }^{2}$ Central Scientific Research Laboratory, Northern State Medical University, Arkhangelsk, Russia; ${ }^{3}$ Research Institute of Internal and Preventive Medicine - Branch of ICandG SB RAS, Novosibirsk, Russia; ${ }^{4}$ Novosibirsk State Medical University, Ministry of Health of Russia, Novosibirsk, Russia; ${ }^{5}$ Department of Community Medicine, Arctic University of Norway, UiT, Tromsø, Norway

\subsection{6/jech-2018-SSMabstracts.177}

Background Common mental disorders (anxiety and depression) are important public health concerns worldwide. There is very little evidence about the risk factors for these in Russia despite high rates of associated markers: suicide and alcohol-attributable mortality. The aim of this study was to investigate the associations between common mental disorders and socio-demographic factors in the Russian adult population.

Methods Data were from two population-based cross-sectional surveys conducted in 2016-17 among men and women aged 35-69 years old resident in the Russian cities of Arkhangelsk and Novosibirsk as part of the International Project on Cardiovascular Disease in Russia (IPCDR). Participants were recruited and interviewed in their homes. Information collected included questions on socio-demographic factors (marital status, education, employment and financial status). Depression was defined as a score of $\geq 10$ on the PHQ-9 instrument and anxiety as a score of $\geq 10$ on the GAD-7 instrument. Financial status was on a 5-point scale from not enough money for food to no financial constraints. Logistic regression was used to estimate effects of socio-demographic factors on anxiety and depression adjusting for age, sex and city and then for all other socio-demographic variables. Volume of alcohol consumed per year and problem drinking defined as CAGE score $\geq 2$ were entered into the models at the last step in order to assess the effect on the associations of interest.

Results After adjustment for all socio-demographic variables depression was more prevalent among women (OR 1.93 95\% CI 1.53, 2.44), those who were divorced or separated compared to married (OR $1.4695 \% \mathrm{CI} 1.10,1.92$ ), and not in regular employment (OR 1.49 95\% CI 1.18, 1.88). There was a monotonic increase in risk of depression across the 5 categories of financial status from poorest to most affluent ( $\mathrm{p}$-value trend $\leq 0.001$ ), with an OR $>6$ from bottom vs top category. Anxiety was more prevalent in women (OR 1.93 95\% CI 1.42, 2.62). As with depression financial status also showed a strong inverse trend in risk $(\mathrm{p}<0.001)$. Education was inversely associated with depression and anxiety after adjustment for age, sex, and city but not after adjustment for other socio-demographic variables. The pattern of results remained similar after additional adjustment for alcohol use.

Conclusion Degree of financial hardship was strongly associated with symptoms of anxiety and depression even after adjustment for education, employment status and alcohol use. These findings underline the importance of considering social circumstances in addressing poor mental health.

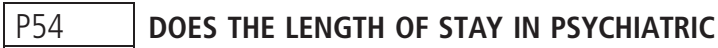 INPATIENT UNITS AFFECTS THE SOCIAL INTEGRATION OF SEVERE MENTALLY-ILL PATIENTS? A STUDY IN FIVE EUROPEAN COUNTRIES}

P Smith*, P Nicaise, V Lorant. Institute of Health and Society, Université Catholique de Louvain, Brussels, Belgium

\subsection{6/jech-2018-SSMabstracts. 178}

Background During the last three decades, in high-income countries, a transition from institutional psychiatric care to community care and a reduction in the hospital length of stay has taken place to provide community care and promote patients' social integration. However, the impact of length of stay in psychiatric inpatient unit on the different dimensions of patients' social integration remains unclear. The aim of this study is to determine whether longer length of stay in psychiatric inpatient unit decreases the social integration of Severe Mentally-Ill (SMI) patients.

Methods Within the European COFI study (prospective cohort study), data were collected for 7302 SMI patients hospitalised in 2015 in the UK, Italy, Germany, Poland and Belgium. Social integration was measured using the SIX index in baseline and at one year of follow-up. The SIX index includes the following dimensions of social integration: employment, housing, living situation and contacts with friends. Correlation and regressions models were performed to test the association between length of stay in psychiatric inpatient unit and patients' social integration.

Results The average score of social integration of SMI patients in baseline was $3.8 / 6 \quad(\mathrm{SD}=1.39)$ and decreased by -0.14 $(S D=1.29)$ one year later. We found a small but significant negative correlation between length of stay in hospital and social integration of patients at one year $(\mathrm{r}=-0.04, \mathrm{p}=0.03)$. This association remained significant when adjusting for patient characteristics and hospitals as a random intercept. At one year, the most correlated dimensions of social integration with length of stay were housing $(\beta=-6.3, p<0.0001)$ and employment $(\beta=-1.8, \mathrm{p}=0.01)$.

Conclusion This study supports the importance of policies and interventions to reduce the length of stay in psychiatric inpatient unit for SMI patients to preserve their social integration. Housing and employment are the main dimensions of social integration negatively impacted by length of stay. Therefore, special attention must be given to help SMI patients to find and retain housing and employment during psychiatric hospitalisations. 